\title{
The shock of it: sympathetic activation promotes hair greying, and other updates on recent autonomic research
}

\author{
Mitchell G. Miglis ${ }^{1} \cdot$ Nicholas Larsen $^{1} \cdot$ Srikanth Muppidi ${ }^{1}$
}

Received: 4 November 2020 / Accepted: 5 November 2020 / Published online: 19 November 2020

(c) Springer-Verlag GmbH Germany, part of Springer Nature 2020

Keywords Stress $\cdot$ Sympathetic $\cdot$ Vagus $\cdot$ Recording $\cdot$ Grey $\cdot$ Hair

\section{The shock of it: sympathetic activation promotes hair greying}

It is well-known in popular culture that stress causes white hair. Anecdotal evidence dating back to the 1800s suggests this could be indeed the case [1], however the pathophysiological mechanisms have been poorly understood, until now. In the most comprehensive study thus far on hair greying, Zhang and colleagues studied the effect that stress has on tissue regeneration in melanocyte stem cells. The results were recently published in Nature [2]. The investigators used several mouse strains in their study that were balanced based on sex and hair cycle. In the first experiment, mice were exposed to three different types of stress and the number of unpigmented white hair was quantified over time. One of the stressors was nociception, which was achieved by inflicting pain through injectoin of resiniferatoxin, an analogue of capsaicin. The results showed that all three stressors led to increased numbers of unpigmented white hairs over time, with nociception having the strongest effect. All mice subjected to stress had an increase in serum corticosterone and norepinephrine. When mice received the opioid analgesic buprenorphine along with resiniferatoxin, catecholamines did not increase and white hair cells did not develop.

To understand how stress effected the melanocyte lineage, resiniferatoxin was injected into mice during the anagen and telogen stage of the cell cycle. This resulted in a selective loss of melanocyte stem cells, however differentiated melanocytes were not affected and continued to produce pigment. When the next round of anagen started, differentiated melanocytes were not produced and unpigmented

Srikanth Muppidi

muppidis@stanford.edu

1 Department of Neurology and Neurological Sciences, Stanford University, Palo Alto, CA, USA hairs emerged. This suggests that melanocyte stem cells are very sensitive to stress, while differentiated melanocytes and melanin synthesis are not directly affected. As a follow-up experiment, the investigators evaluated how stress induces melanocyte stem cell loss. Resiniferatoxin injection into immunodeficient mice did not result in hair greying, suggesting that hair greying is not necessarily immunemediated. To determine whether stress-induced circulating factors have a role in the loss of melanocyte stem cells, the authors depleted glucocorticoid receptors and blocked the $\beta 2$-adrenergic receptor (Adrb2). Depletion of glucocorticoid receptors resulted in ongoing hair greying in response to stress, while $\beta 2$-adrenergic receptor (Adrb2) blockade prevented hair greying in response to stress. Furthermore, injection of norepinephrine in the absence of stress was sufficient to cause hair greying. These results suggest that the expression of the $\beta 2$-adrenergic receptor in melanocyte stem cells is essential for stress-induced hair greying.

To identify the source of norepinephrine under stress, resiniferatoxin was injected into adrenalectomized mice. These mice continued to develop grey hairs in response to stress, suggesting that the adrenal glands were not the source of catecholamine release. The authors found that stress caused a robust induction of FOS, a reliable marker of neuronal activity, in the cell bodies of sympathetic neurons. Sympathetic nerves were then chemically ablated and norepinephrine was blocked in sympathetic nerve terminals, which prevented resiniferatoxin-induced hair greying and loss of melanocyte stem cells. Finally, the authors activated the sympathetic nervous system using Gq-DREADD, which resulted in the loss of melanocyte stem cells and caused hair greying. Collectively, these data suggest that norepinephrine secreted from sympathetic nerve terminals mediates the effect of stress on melanocyte stem cells. Using a mouse strain in which melanocyte stem cells can be traced by their membrane expression of GFP, the authors found that injection of 
resiniferatoxin resulted in differentiation and migration of melanocyte stem cells into the epidermis and dermis, areas that are normally devoid of pigments. In stressed mice, the investigators found upregulation of several cell-cycle regulators, genes involved in promoting melanogenesis and genes involved in melanocyte stem cell proliferation, differentiation and migration. These data suggest that melanocyte stem cells upregulate their proliferation and differentiation programs after stress.

The primary limitation of this study is that it was conducted in a mouse model, and its translatability to humans still needs to be investigated. Nonetheless, this is the first comprehensive study to demonstrate that stress leads to the depletion of melanocyte stem cells, resulting in permanent damage to tissue regeneration via activation of the sympathetic nervous system. These findings suggest that the sympathetic nervous may affect a variety of processes in development and tissue maintenance. Future studies should focus on the degree to which stress is associated with the loss of melanocyte stem cells that occurs in natural aging.

\section{Intraneural vagus nerve recording: are we there yet?}

Vagal nerve stimulation is a treatment option for myriad diseases including epilepsy and depression and may also hold promise for the treatment of autoimmune diseases such as rheumatoid arthritis. This likely reflects the prominent role that autonomic pathways play in diverse physiological and pathological states of immune function. Most autonomic physicians are well versed in the analysis of heart rate variability (HRV) as a marker of cardiovagal function, as commonly performed in standardized autonomic laboratories. Researchers have also demonstrated the ability to record directly from the vagus nerve with ultrasound-assisted microneurography, a topic we have previously covered in this editorial. However, to date, we do not have intraneural recordings from the vagus nerve, a possible breakthrough as the field moves towards further measures to improve or alter vagal nerve function. HRV remains an important variable in many diseases, with increasing evidence to suggest that preserved HRV is associated with longer lifespan [3]. This particular feature has even led to unique projects, such as the Palo Alto longevity project (https://paloaltoprize.com) to improve HRV as surrogate marker of longevity.

For these reasons, we urgently need data from direct vagal nerve recording in humans, even though technical aspects of accessing the vagal nerve limit accessibility. Recently, researchers from the University of Michigan published their work using multi-channel high-density intraneural carbon fiber microelectrode array (CFMA) with ultra-small electrodes to record directly from very small nerves such as the vagus [4]. In this study, the authors inserted 16 carbon fibers in the cervical vagus nerve of anesthetized rats. They were able to observe intraneural recordings in most of their experiments. They were able to record periodic burst frequency at approximately $40 \mathrm{~Hz}$, a frequency similar to the rats' respiratory rate. When the anesthetized rats' breathing frequency was reduced to $20 \mathrm{~Hz}$, the burst repetition rate also reduced to a similar frequency. Since these recordings were from 1 or 2 out of 16 functional channels, it was felt that these reflect direct vagal nerve recording and not surrogate measures of respiratory rate. Additionally, the researchers were able to obtain the vagal nerve firing rates with various experiments involving blood glucose modulation. For this part of the study, animals were injected with intraperitoneal glucose, insulin, 2-deoxy-D-glucose or saline and intraneural vagal firing patterns were recorded. Although individual firing rates did not correlate with blood glucose modulation, there were clusters of firing rates in the vagus nerve that were reduced with administration of glucose, as well as additional signal propagation in an afferent direction. All of this suggests a clear change in vagal firing with the intervention.

This study-utilizing direct action potential recording from the cervical vagus nerve of rats with a multi-channel CFMA - was able to demonstrate that recording intraneural action potential recordings is feasible. This proof of concept study will advance the field, but it is unlikely that we will be able to perform similar studies in humans without further experiments. These researchers were likely able to record from some but not all of the 16 electrodes, which could be related to the technical difficulty accessing the very small diameter vagus nerve of the rat. We hope this initial work will lead to additional animal studies and eventually studies in humans, with future applications in vagal nerve stimulation and other therapeutics for autonomic disorders.

\section{A novel neuroanatomical pathway explaining psychosomatic stress}

Since the seminal experiments by Walter Cannon, the importance of sympathetic activation in the stress response is well accepted. The classic "fight or flight" response includes tachycardia, hypertension and hyperhidrosis. These physiological responses are evolutionarily conserved mechanisms to prepare our body to fight against imminent dangers, or to run away quickly. Another key response is an increase in body temperature. Interestingly, emotional stress can cause psychogenic fever in many mammalian species, from rodents to humans. However, we are far from a complete understanding of the neurobiological basis of stress.

In humans, the stress response produced by emotional or psychological triggers often involves an understanding of complicated situations (i.e., the future, the past, etc.), 
which requires input from the brain cortical regions, which are involved in memory, cognitive, and emotional processes.

In a recent study published in Science, Katoaka and colleagues aimed to identify the cortical regions that transmit stress signals to the dorsomedial hypothalamus (DMH), a key central structure in stress responses [5]. To do so, the authors used retrograde neural tracers (cholera toxin b-subunit) which they injected into the DMH, to look for neurons that link with a heat-generating circuit. They found that only one, little studied, region of the cortex was strongly labeled by the tracer. This relatively unknown region, called the $d o r$ sal peduncular cortex and dorsal taenia tecta (DP/DTT), is highly active in rats in the wake of social defeat (a hostile interaction in which the animal has lost a fight with another, dominant rat).

To examine the role of this region in stress responses, the authors impaired its connection to the DMH in three ways. They blocked the activity throughout the DP/DTT using a chemical inhibitor; they used a virus to kill cells projecting from the DP/DTT to the DMH; and they used a sophisticated genetic approach to inhibit activity specifically in the projections that DP/DTT neurons send to the DMH. In each case, their intervention reduced stress-induced hyperthermia.

By contrast, artificial activation of the neuronal projections between the two regions elicited a battery of responses, including increases in heart rate, blood pressure, and heat production in brown fat. The authors provided evidence that the DP/DTT neurons send excitatory signals to the DMH, and demonstrated that the projections from the DP/DTT terminate close to the DMH cells that, in turn, project to the rostral medullary raphe.

Taken together, Kataoka and colleagues' experiments support the idea of a DP/DTT-DMH-rostral medullary raphe-brown fat circuit for heat production in response to stress. This represents an important psychosomatic integrator through which stress and emotions affect the autonomic nervous system. Indeed, the authors postulate that this DP/ DTT-DMH pathway might serve as a potential therapeutic target for psychosomatic or functional disorders, especially as this pathway does not contribute to basal homeostasis and thus would not affect the autonomic reflexes crucial to survival. The main critique of this study, as in our other updates in this issue, is the applicability of the rat model to human physiology. Also, while every possible stressor, from physical pain to present or anticipated psychologically uncomfortable situations (e.g., an imminent death in the family, an ongoing pandemic, or an adverse result in the general election), can find their way to the DP/DTT, it remains unclear how different stressors are programmed in the DP/ DTT, whether the responses of the DP/DTT to stressors are influenced by past experiences (presumably they are), and whether abnormalities in DP/DTT cells or pathways could mediate exaggerated physiological responses to stress (e.g., psychosomatic disorders, anxiety, panic disorders, posttraumatic stress disorder). Future studies using electrophysiological or optical recordings of the DP/DTT cells should help to address these remaining questions.

Nonetheless, these findings illustrate a novel neuroanatomical pathway that may serve an important role in stress signaling; responses that in moderation are critical for survival; however, in excess may lead to many disease states. Further studies-ideally in humans-are necessary to confirm this pathway and explore its potential as a therapeutic target in patients with functional syndromes with exaggerated responses to stress, so frequently seen in the autonomic clinic.

Funding None.

\section{Compliance with ethical standards}

Conflict of interest The author(s) declare that they have no conflicts of interest.

\section{References}

1. Ephraim AJ (1959) On sudden or rapid whitening of the hair. AMA Arch Dermatol 79:228-236. https://doi.org/10.1001/archd erm.1959.01560140090013

2. Zhang B, Ma S, Rachmin I et al (2020) Hyperactivation of sympathetic nerves drives depletion of melanocyte stem cells. Nature 577:676-681. https://doi.org/10.1038/s41586-020-1935-3

3. Hernández-Vicente A, Hernando D, Santos-Lozano A et al (2020) Heart rate variability and exceptional longevity. Front Physiol 11:566399. https://doi.org/10.3389/fphys.2020.566399

4. Jiman AA, Ratze DC, Welle EJ et al (2020) Multi-channel intraneural vagus nerve recordings with a novel high-density carbon fiber microelectrode array. Sci Rep 10:1-13. https://doi. org/10.1038/s41598-020-72512-7

5. Kataoka N, Shima Y, Nakajima K, Nakamura K (2020) A central master driver of psychosocial stress responses in the rat. Science 367(6482):1105-1112. https://doi.org/10.1126/science.aaz4639 